\title{
Single-Walled Carbon Nanotube-Polymer Composite Thin Film for Flow Sensor Application
}

\author{
Hui Cao ${ }^{1,2}$, Qiang Lv ${ }^{1,2}$, Han Yan ${ }^{2,3}$, Xiaohui Song ${ }^{5}$, \\ Xiaobin $\mathrm{Luo}^{2,4}$, Zhiyin Gan ${ }^{1,2, *}$ and Sheng Liu ${ }^{1,2, * *}$ \\ ${ }^{1}$ School of Mechanical Science and Engineering, \\ Huazhong University of Science and Technology, Wuhan, China \\ 2Institute of Microsystems, WuHan National Laboratory for Optoelectronics, Wuhan, China \\ ${ }^{3}$ Department of Electronic Science and Technology, \\ Huazhong University of Science and Technology, Wuhan, China \\ ${ }^{4}$ School of Energy and Power Engineering, \\ Huazhong University of Science and Technology, Wuhan, China \\ ${ }^{5}$ Research Institute of Micro/Nano Science and Technology, \\ Shanghai Jiao Tong University, Shanghai, China
}

(Received January 9, 2009; accepted April 22, 2009)

Key words: carbon nanotube, composite thin film, flow sensor

We report a fabrication method for a composite thin-film flow sensor including a single-walled carbon nanotube (SWCNT) network and polydimethylsiloxane (PDMS), which is immersed in deionized water and $\mathrm{NaCl}$ solution. The morphology of SWCNTs on the surface of the composite thin film is characterized by scanning electron microscopy. The induced voltage generated along the direction of the flowing liquid depends significantly on the liquid concentration and flow velocity. Since the SWCNTs are coated with polymer chains and fixed into the PDMS matrix, the $I-V$ curves of the composite thin film are completely coincident before and after several flow velocity measurements, and the repeated flow-induced voltage experiment shows that the composite thin film has a reliable electrical characteristic and a wide potential of device application.

\section{Introduction}

A carbon nanotube (CNT) is a type of carbon allotrope, which was discovered in 1991 by a Japanese scientist, Iijima. ${ }^{(1)}$ It's thermal, mechanical, chemical stability, and large current-carrying capacities make it attractive for application in electrical interconnects, ${ }^{(2,3)}$ field-effect transistors, ${ }^{(2,4)}$ cold cathode field emitters, ${ }^{(5,6)}$ and sensors. ${ }^{(7,8)}$ CNTs can exhibit good conductor and semiconductor behaviors owing to their unique structures. ${ }^{(9)}$ One of the primary hurdles in developing such novel CNT devices is the absence of a reliable method of fabrication.

${ }^{*}$ Corresponding author: e-mail: ganzhiyin@126.com

${ }^{* *}$ Corresponding author: e-mail: victor_liu63@126.com 
In a recent remarkable experiment, Ghosh and coworkers ${ }^{(10,11)}$ observed that the flow of an ion-rich liquid, such as deionized water or $\mathrm{HCl}$ solution, through bundles of metallic single-walled carbon nanotube (SWCNT) networks generates a voltage in the nanotube along the direction of the liquid flow when CNT films are immersed in flowing liquids. Liu et al. ${ }^{(12)}$ found that multiwalled carbon nanotubes (MWCNTs) also generate an electric voltage when $\mathrm{NaCl}$ solution flows through the nanotube network (NTN). These studies indicate that the voltage generation depends on the polar nature of the liquid. These studies reveal the potential application of a CNT flow sensor. Since the nanotubes are deposited on the substrate without being fixed, the CNTs are washed away by the liquid and the performance of the sensor is unstable. In addition, the unfixed CNTs relatively reduce the movement between fluid and NTN.

In this study, we provide a synthetic process for fabricating a SWCNT network/ polydimethylsiloxane (PDMS) composite thin film-based flow sensor. The SWCNTs are coated with PDMS polymer chains and fixed into the PDMS matrix to prevent the washing away of SWCNTs by liquid. The features of the electric voltage generated by ion-rich liquid are similar to those observed in previous studies.

\section{Experiments}

The SWCNTs used in this study were supplied by Chengdu Organic Chemicals Company, with an average diameter of about $2 \mathrm{~nm}$, an average length of about $30 \mu \mathrm{m}$, and a purity above $90 \mathrm{wt} \%$. The PDMS was a transparent flexible insulator and was supplied by Dow Corning Company.

The SWCNT suspensions were prepared by sonicating the raw materials in dimethylformamide (DMF, 99.9\%, J. T. Baker) for $8 \mathrm{~h}$ at a power level of $80 \mathrm{~W}$ with subsequent centrifugation at $16,000 \mathrm{rpm}$ for $1 \mathrm{~h}$. The upper $10 \%$ of the supernatant was then carefully decanted. The resulting CNT suspension had a concentration of approximately $20 \mathrm{mg} / \mathrm{L}$. The suspension was then ultrasonically treated for $2 \mathrm{~h}$ to sufficiently disperse the SWCNTs. The CNT networks ${ }^{(13)}$ were then obtained by vacuum filtration ${ }^{(14)}$ using a poly-tetrafluoro-ethylene (PTFE) filter with an average pore size of $220 \mathrm{~nm}$, as shown in Fig. 1(a). To enhance the degree of the distribution uniformity of the CNT film, the vacuum level was controlled to extend the filtration time. ${ }^{(14)}$

The CNT film formed on the surface of the filter (Fig. 1(a)) was placed in a mould (Fig. 1(b)) and transferred to a substrate by direct PDMS molding (Fig. 1(c)). In this process, the PDMS was poured onto the filter surface and cured at $60^{\circ} \mathrm{C}$ for $8 \mathrm{~h}$ in a lowvacuum oven (5 Pa). After curing, the filter membrane was removed (Fig. 1(d)) and the CNT/PDMS hybrid thin film was formed on the PDMS substrate. The metal electrodes, $10 \AA$ of chromium (adhesion layer) and $200 \AA$ of gold, were deposited onto the surface of the CNT/PDMS composite thin film (Fig. 1(d)) by thermal evaporation. ${ }^{(15)}$ Finally, the cured PDMS cover with a square cross-sectional flow channel was treated in an oxygen plasma for $1 \mathrm{~min}$ to form oxidized PDMS ${ }^{(16)}$ and then bonded to the PDMS substrate (Fig. 1(e)). A schematic of the flow sensor is shown in Fig. 1(f). The CNT/PDMS composite thin film was placed at the center of the flow channel with an inner square side of $2 \mathrm{~mm}$ and a length of $60 \mathrm{~mm}$ to prevent any turbulent flow caused by the expansion of the flow 


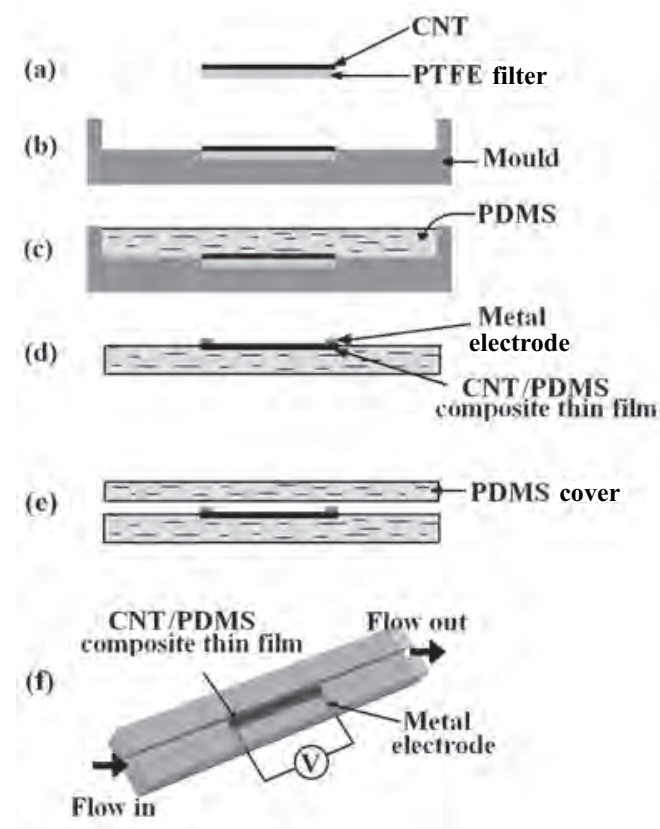

Fig. 1. Process of fabricating flow sensor: (a) SWCNT network is formed on PTFE filter membrane by vacuum filtration. (b) PTFE filter membrane with SWCNT network is placed in the mould. (c) PDMS is poured into the mould and cured. (d) Filter membrane is removed, the SWCNTs are transferred to PDMS, and then the metal electrodes of $\mathrm{Cr}$ and $\mathrm{Au}$ are deposited on the CNT/PDMS composite thin film by thermal evaporation. (e) PDMS cover is bonded to the PDMS substrate. (e) Schematic of flow sensor.

at the inlet of the channel. The flow velocity in the channel was controlled using a liquid flow controller (L23V02, Bronkhorst, Netherlands). The flow-induced voltage along the CNTs was measured using a digital multimeter/data acquisition system (Medel 2007, Keithley, USA).

Two liquids, namely, deionized water (DI water) and aqueous $\mathrm{NaCl}$ solution, were allowed to flow over the NTN/PDMS composite thin film in our experiments. Experimental conditions, such as liquid velocity and $\mathrm{NaCl}$ solution concentration were varied and the voltage between two electrodes was measured repeatedly.

\section{Results and Discussion}

To investigate the morphology of SWCNTs in the NTN/PDMS composite thin film, scanning electron microscopy (SEM, Sirion 200, FEI, Holand) was used to obtain the microstructure of the thin film (Fig. 2). Since it was fabricated by vacuum filtration, the film was composed of highly entangled SWCNT bundles held together by a van der 


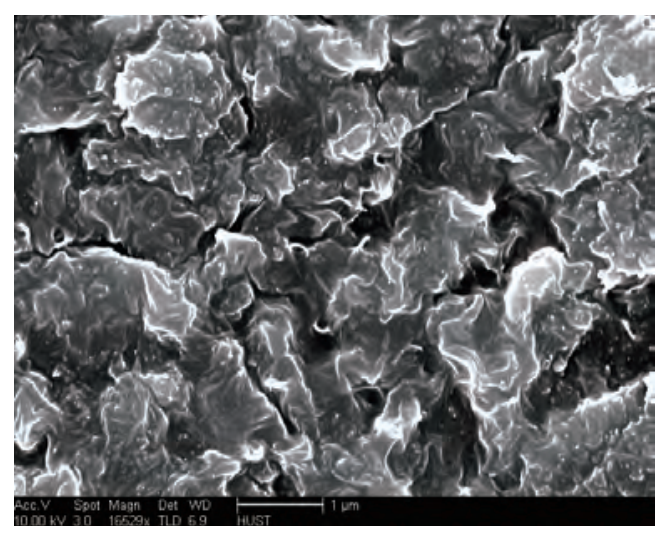

(a)

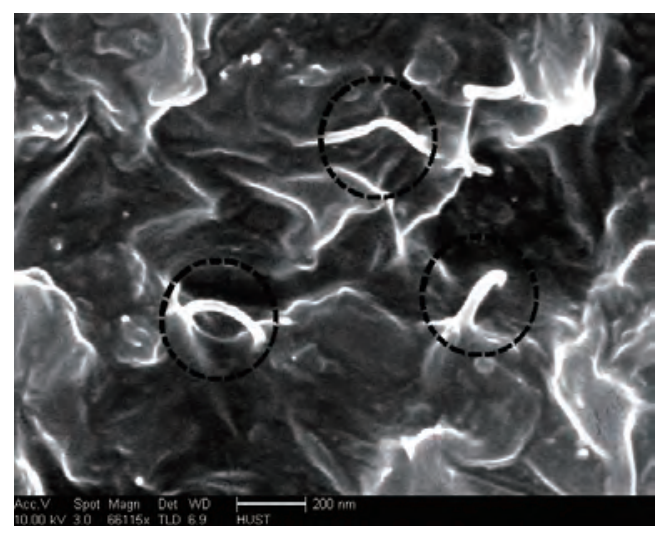

(b)

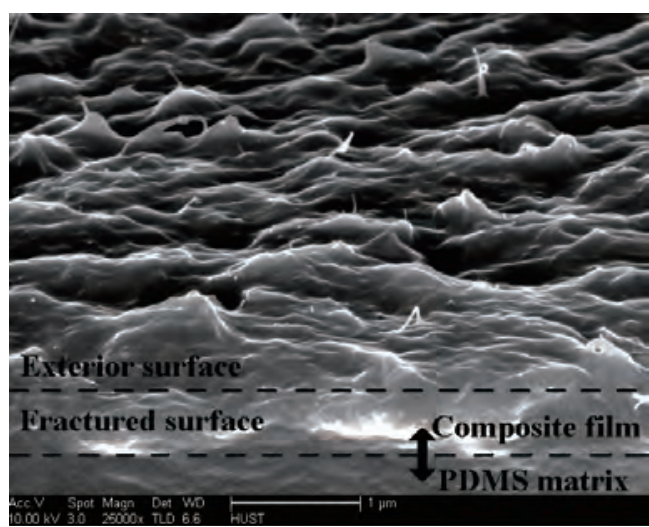

(c)

Fig. 2. SEM image of NTN/PDMS composite thin film: (a) Surface of NTN/PDMS composite thin film. (b) Highly magnified image of (a). (c) Surface and cross section of composite thin film.

Waals attraction force, and the SWCNTs appeared randomly oriented and uniformly

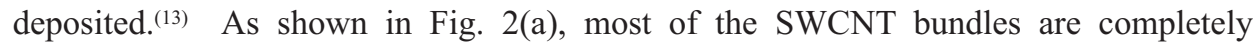
embedded in the cured PDMS matrix, and some of them lie near the surface protruding above the composite film. Figure 2(b) shows the highly magnified image in Fig. 2(a). It shows that the SWCNTs are coated with PDMS, suggesting that the SWCNTs are bonded to PDMS polymer chains and fixed during PDMS curing. The outer diameter of the tubes is consistent for the different morphologies and is on the order of approximately less than $50 \mathrm{~nm}$. The thickness of the NTN/PDMS composite films is less than $1 \mu \mathrm{m}$, as shown in Fig. 2(c). 
The dimensions of the NTN/PDMS composite thin film used in the flow sensor are about $20 \times 2 \mathrm{~mm}^{2}$. The Reynolds number $(R e)(\sim 30$ for a velocity of $10 \mathrm{~mm} / \mathrm{s}$, DI water $)$ of the chamber is much less than the critical value of 2000 for the turbulent flow in a pipe. Thus, the flow at the center of the chamber is laminar. There was an original voltage of about $3 \mathrm{mV}$ when the flow sensor was immersed in solution, which was explained by Ghosh et al. ${ }^{(10)}$ as an electrochemical potential difference at the interface of the SWCNT bundles with the metal electrodes. The difference between the measured and offset voltages is considered to be due to a flow-induced voltage.

For individual SWCNTs, a theoretical investigation method for electrical current generation has been proposed by Kral and Shapiro. ${ }^{(17)}$ One mechanism of such current generation is the transfer of momentum from the flowing liquid molecules to the acoustic phonons in the nanotube as the phonon quasi-momentum, which in turn drags free carriers into the nanotube. The other mechanism involves the direct scattering of free carriers from the fluctuating Coulombic fields of the ions or polar molecules in the flowing liquid.

The relationship between the flow-induced voltage and the flow rate of DI water or $\mathrm{NaCl}$ solution is shown in Fig. 3. The voltage increases with increasing flowing velocity and saturates slowly. This sublinear dependence on the flow velocity indicates a pulsating asymmetric ratcheting mechanism. ${ }^{(10)}$ The voltage generated under the condition of DI water is much smaller than that generated under the condition of $\mathrm{NaCl}$ solution. When $\mathrm{NaCl}$ solution with a concentration of $20 \mathrm{wt} \%$ flows over the sensor at a rate of $3 \mathrm{~mm} / \mathrm{s}$, the flow-induced voltage increases to $5.83 \mathrm{mV}$.

Figure 3 also shows the relationship between the flow-induced voltage and concentration of $\mathrm{NaCl}$ solution. When $\mathrm{NaCl}$ solutions with concentrations of 5,10 , and $20 \%$ flow over the sample at a rate of $3 \mathrm{~mm} / \mathrm{s}$, the induced-voltage reaches 5.71 , 5.79 , and $5.83 \mathrm{mV}$, respectively. The voltage induced by the liquid flow depends on the

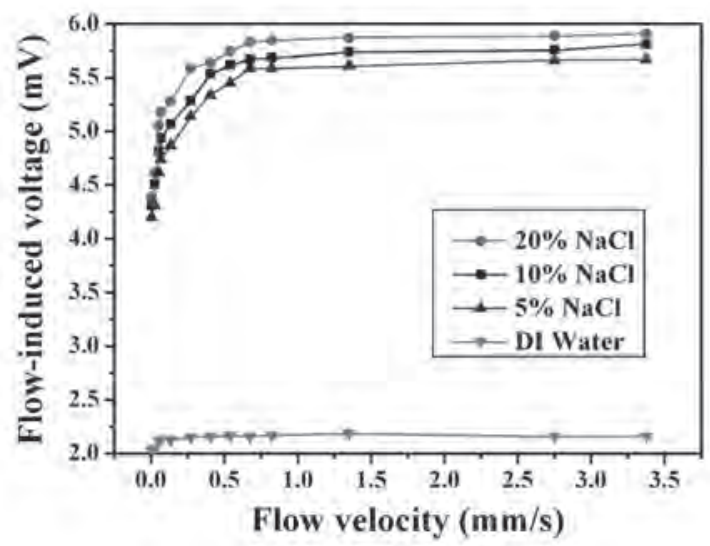

Fig. 3. Flow-induced voltage versus flow velocity. 
concentration of the flowing liquid, suggesting that the mechanism involving fluctuating Coulombic fields dominates the phonon-mediated electron drag. ${ }^{(10)}$

To investigate the stability of this NTN/PDMS composite thin film, several experiments were conducted. Figure 4(a) shows the flow-induced voltage measured repeatedly at the same velocities of $0.027,0.0675$, and $0.675 \mathrm{~mm} / \mathrm{s}$ with $20 \mathrm{wt} \% \mathrm{NaCl}$ solution, and the observed changes correspond to $0.8,1.2$, and $0.7 \%$, respectively. When a DC voltage was applied between two electrodes, the film exhibited a linear relationship

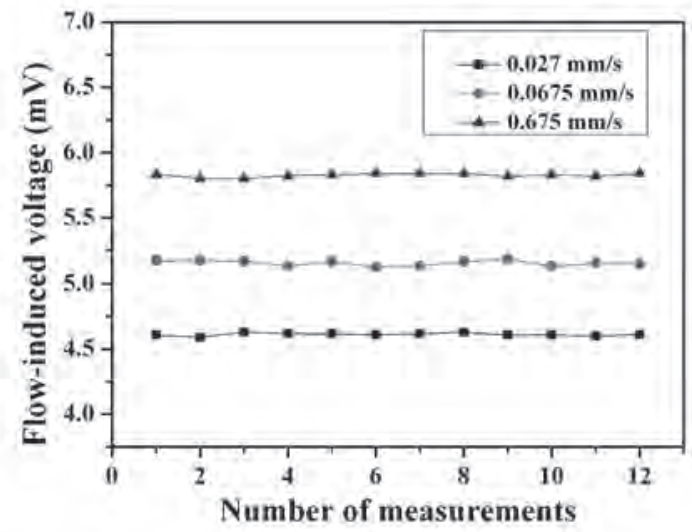

(a)

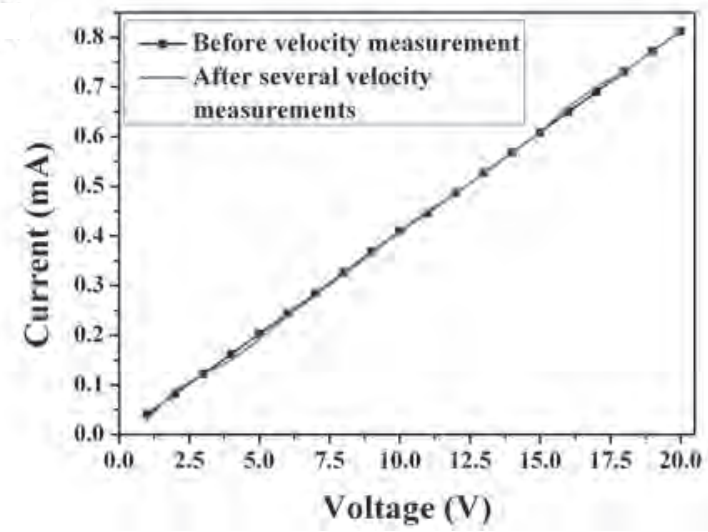

(b)

Fig. 4. (a) $I-V$ curve of NTN/PDMS composite thin film before and after velocity measurement. (b) Repeat voltage measurement at flow rates of $0.027,0.0675$, and $0.675 \mathrm{~mm} / \mathrm{s}$ with $20 \mathrm{wt} \% \mathrm{NaCl}$ solution. 
between voltage and current. The $I-V$ curve of the composite thin film was measured before and after several velocity measurements. As shown in Fig. 4(b), the two curves are almost coincident with each other, and the maximum change rate is about $0.197 \%$ at $2.5 \mathrm{~V}$. These results show a stable electrical characteristic in the composite thin film and make it suitable for flow sensing.

\section{Conclusions}

Experimental results show that the flow-induced voltage could be generated using NTN/PDMS composite thin films immersed in flowing liquids. The flow-induced voltage depends on the flow rate and concentration of the liquids. The SEM images of this thin film show that SWCNTs are coated with polymer chains and fixed in the PDMS matrix. The experimental results of repeated measurement and the $I-V$ curve indicate that this NTN/PDMS composite is highly stable, which could lead to flow-induced nanotube flow sensors of practical significance. This sensor can be scaled down to dimensions on the order of micrometers, which makes it suitable for very small liquid volumes.

\section{Acknowledgments}

Our team is grateful to the School of Mechanical Science \& Engineering and WuHan National Laboratory for Optoelectronics for supporting this work. This work was supported by the National High Technology Research and Development "863" Program of China (No. 007AA04Z348) and the China Postdoctoral Science Foundation (No. 20080440139).

\section{References}

1 S. Iijima: Nature 354 (1991) 56.

2 S. B. Lee, K. B. K. Teo, G. A. J. Amaratunga, W. I. Milne, M. Chhowalla, D. G. Hasko and H. Ahmed: J. Vac. Sci. Technol. B 21 (2003) 996.

3 Y. H. Lee, Y. T. Jang, C. H. Choi, E. K. Kim, B. K. Ju, D. H. Kim, C. W. Lee and S. S. Yoon: J. Appl. Phys. 91 (2002) 6044.

4 M. Ahlskog, R. Tarkiainen, L. Roschier and P. Hakonen: Appl. Phys. Lett. 77 (2000) 4037.

5 I. T. Han, H. J. Kim, Y. J. Park, N. Lee, J. E. Jang and J. W. Kim: Appl. Phys. Lett. 81 (2002) 2070.

6 Y. Huh: Chem. Phys. Lett. 375 (2003) 388.

7 E. S. Snow, F. K. Perkins, E. J. Houser, S. C. Badescu and T. L. Reinecke: Science 307 (2005) 1942.

8 Z. Hou, D. Xu and B. Cai: Appl. Phys. Lett. 89 (2006) 213502.

9 M. S. Dresselhaus: Nature 391 (1998) 19.

10 S. Ghosh, A. K. Sood and N. Kumar: Science 299 (2003) 1042.

11 S. Ghosh, A. K. Sood, S. Ramaswamy and N. Kumar: Phys. Rev. B 70 (2004) 205423.

12 J. W. Liu, L. M. Dai and J. W. Baur: J. Appl. Phys. 101 (2007) 064312.

13 A. G. Rinzler, J. Liu, H. Dai, P. Nikolaev, C. B. Huffman, F. J. Rodriguez-Macias, P. J. Boul, A. H. Lu, D. Heymann, D. T. Colbert, R. S. Lee, J. E. Fischer, A. M. Rao, P. C. Eklund and R. E. Smalley: Appl. Phys. A 67 (1998) 29. 
14 Z. C. Wu, Z. H. Chen, X. Du, J. M. Logan, J. Sippel, M. Nikolou, K. Kamaras, J. R. Reynolds, D. B. Tanner, A. F. Hebard and A. G. Rinzleret: Science 305 (2004) 27.

15 D. A. Bernards, T. Biegala, Z. A. Samuels, J. D. Slinker and G. G. Malliaras: Appl. Phys. Lett. 84 (2004) 3675.

16 D. R. Reyes, D. Lossifidis, P. A. Auroux and A. Manz: Anal. Chem. 74 (2002) 2623.

17 P. Kral and M. Shapiro: Phys. Rev. Lett. 86 (2001) 131. 